\title{
Assessing auditory distance perception using perceptually directed action
}

\author{
JACK M. LOOMIS \\ University of California, Santa Barbara, Califormia \\ ROBERTA L. KLATZKY \\ Carnegie Mellon University, Pittsburgh, Pennsylvania \\ and \\ JOHN W. PHILBECK and REGINALD G. GOLLEDGE \\ University of California, Santa Barbara, California
}

\begin{abstract}
Three experiments investigated auditory distance perception under natural listening conditions in a large open field. Targets varied in egocentric distance from 3 to $16 \mathrm{~m}$. By presenting visual targets at these same locations on other trials, we were able to compare visual and auditory distance perception under similar circumstances. In some experimental conditions, observers made verbal reports of target distance. In others, observers viewed or listened to the target and then, without further perceptual information about the target, attempted to face the target, walk directly to it, or walk along a two-segment indirect path to it. The primary results were these. First, the verbal and walking responses were largely concordant, with the walking responses exhibiting less between-observer variability. Second, different motoric responses provided consistent estimates of the perceived target locations and, therefore, of the initially perceived distances. Third, under circumstances for which visual targets were perceived more or less correctly in distance using the more precise walking response, auditory targets were generally perceived with considerable systematic error. In particular, the perceived locations of the auditory targets varied only about half as much in distance as did the physical targets; in addition, there was a tendency to underestimate target distance, except for the closest targets.
\end{abstract}

Spatial hearing is concerned with the localization of sound sources and reflecting surfaces. In this paper, our focus is on the localization of sound sources. The egocentric location of a source is usually specified in these following coordinates: azimuth (compass direction with respect to the facing direction of the head), elevation, and distance. The vast majority of studies on the localization of sources has been concerned with directional hearing; only a few studies have dealt with auditory distance perception. Now, however, the emerging technology of virtual acoustics is promoting considerable interest in the topic. Virtual acoustic displays have considerable promise as interfaces between humans and computers in which "virtual" or "spatialized" sound is used to represent real and virtual environments and abstract data structures (Begault, 1994; Carlile, 1996; Durlach, 1991; Gilkey \&

This research was supported by Grant 9740 from the National Eye Institute. Some of these results were presented at the 1994 meeting of the Psychonomic Society, St. Louis, MO. The authors thank Fred Wightman and an anonymous reviewer for comments on an earlier version of this article, and Sue Babey, Sarah Hunter, Patrick Monnier, and Tony Richardson for assistance with the experiments. Correspondence should be addressed to J. M. Loomis, Department of Psychology, University of California, Santa Barbara, CA 93106-9660(e-mail: loomis@ psych.ucsb.edu).
Anderson, 1997; Kendall \& Martens, 1984; Kramer, 1994; Loomis, Hebert, \& Cicinelli, 1990; Wenzel, 1992). Because auditory space is three-dimensional, an optimal display is one that makes use of perceptual variation in distance as well as in elevation and azimuth. Consequently, there is a need to understand how well humans perceive auditory distance, which stimulus cues support such perception, and which distortions of the stimulus, if any, might be effected to promote the desired localization of virtual sounds.

\section{Virtual Acoustic Displays}

The research we are reporting stems from an interest our research group has in using virtual sound to aid in the spatial orientation of the blind. Since the beginning of this project (Loomis, 1985), we have been working toward a personal guidance system for the visually impaired that uses a virtual acoustic display as part of the user interface (Golledge, Loomis, Klatzky, Flury, \& Yang, 1991; Loomis, Golledge, Klatzky, Speigle, \& Tietz, 1994). With such a display, one of our goals has been to have the navigation system lead a blind traveler along specified routes by means of virtual auditory beacons appearing at points along the routes. In addition, the system indicates the positions of important off-route landmarks by having their labels, spoken by speech synthesizer, appear as virtual 
sounds at the appropriate locations within the auditory space of the traveler. We are hopeful that these virtual landmarks will help blind travelers develop better mental representations of the environment. We believe that this is most likely to occur if the traveler perceives the virtual sounds at the intended locations (distances and directions).

Achieving effective distance perception with virtual acoustic displays will probably be some time in coming; just the goal of obtaining externalized sound with earphones has proven a considerable challenge (e.g., Begault, 1992, 1994; Begault \& Wenzel, 1993; Loomis \& Soule, 1996; Wightman \& Kistler, 1989). The question of which stimulus and observer factors are most important in determining the externalization of sound heard through earphones has received considerable attention (Durlach et al., 1992; Loomis et al., 1990; Plenge, 1974; Wenzel, 1992; Wenzel, Arruda, Kistler, \& Wightman, 1993; Wightman \& Kistler, 1989). Despite our early success in achieving "externalized" sound with our virtual display in the laboratory (Loomis et al., 1990), we have been disappointed with the degree of externalization of our portable system, which has employed both a variant of our original display (Loomis et al., 1990) and a commercially available display (the Alphatron from Crytal River Engineering).

Because the virtual displays we have at our disposal produce less externalization than we desire, we have deferred using them to investigate questions about auditory distance perception. Instead, we have conducted research using real sound sources in natural environments. However, as indicated above, the topic of auditory distance perception with normal listening is of great interest in its own right. One such experiment by our group has already been reported (Speigle \& Loomis, 1993) using source distances ranging from 2 to $6 \mathrm{~m}$; more recent experiments using longer distances are reported here.

Research conducted to date on auditory distance perception has been concerned primarily with assessing the stimulus cues that determine perceived distance. A number of potential cues have been identified, some informative about egocentric (absolute) distance to the source and some informative only about the relative distance (ratio of distances) of two sources (Mershon \& King, 1975). The majority of this research has been conducted in indoor laboratories using verbal estimates of the perceived distance of the source.

\section{Static Distance Cues}

There are a number of potential cues to egocentric distance that are available to an observer whose head is stationary. The first of these is sound level. In an anechoic (free-field) environment, sound level falls off by $6 \mathrm{~dB}$ for each doubling of distance (Coleman, 1963). If the observer has independent knowledge about source intensity (i.e., the source is familiar), sound level can serve as an absolute distance cue; if source intensity is not known, then only the relative distances of the source on separate presentations can be known from the ratio of sound pres- sures (Mershon, 1997). Experiments by Mershon and King (1975) and others (cited in Coleman, 1963) have shown that manipulating sound level does influence the observer's judgment of distance. Other cues available to a stationary observer are spectral content and the proportion of direct to reverberant sound; both of these have been shown to influence judgments of perceived distance (Butler, Levy, \& Neff, 1980; Coleman, 1968; Little, Mershon, \& Cox, 1991; Mershon, Ballenger, Little, McMurtry, \& Buchanan, 1989; Mershon \& Bowers, 1979; Mershon \& King, 1975; Sheeline, 1983). Still another potential cue is angular elevation of the source relative to the observer's perceived ear level; the cue relies on the assumption that the source is resting on the horizontal surface plane. The analogous cue for vision (sometimes referred to as height in the field) has been shown to be a strong cue for visually perceived egocentric distance when the target or its support is visibly resting on the ground (Philbeck \& Loomis, 1997). Because sound sources at ground elevation are hardly representative of sources in general, it seems unlikely that angular elevation constitutes a reliable distance cue.

\section{Dynamic Distance Cues}

When an observer translates through space, cues associated with acoustic flow become available. One of these is absolute motion parallax, the changing azimuth of a source (Loomis et al., 1990; Speigle \& Loomis, 1993). Its validity as a distance cue relies on the assumption that the source is stationary. The other motion-related distance cue is referred to as acoustic tau (Ashmead, DeFord, \& Northington, 1995; Guski, 1992; Lee, 1990; Schiff \& Oldak, 1990; Shaw, McGowan, \& Turvey, 1991), the acoustic analog of the optical variable, tau, which specifies time to contact. Acoustic tau is given by $2 I /(d I / d t)$, where $I$ is the intensity of the sound at the observer's ear arriving from the source. Tau specifies the time to collision of an observer approaching the source with a constant closing velocity; source distance is given by the product of acoustic tau and the closing velocity.

Speigle and Loomis (1993) found that the dynamic cues of motion parallax and acoustic tau exerted only a modest influence on observers' judgments of source distance above and beyond the static cues (sound level, spectral cues, and direct/reverberant ratio) that were available in their test environment. Ashmead et al. (1995) found that acoustic tau information improved performance considerably more; in their study, however, sound level was rendered unreliable as a static distance cue by randomly varying source intensity from trial to trial independently of distance. In the present study, we investigated auditory distance perception based only on static cues; in all of the experiments, the observers listened to the sound sources from a fixed location in space.

\section{Perceptually Guided Action}

One difficulty with the concept of perceived egocentric distance is the lack of consensus on how to measure it. Given the longstanding interest in visually perceived 
distance, vision researchers have devoted considerable thought to measurement methods (see Loomis, Da Silva, Fujita, \& Fukusima, 1992, for a brief review of some of these). The methodological issue has received little attention by audition researchers (for an exception, see Mershon, 1997).

The primary method that we employed here used a spatial behavior of the observer that, in connection with vision, has been referred to as visually directed action, open loop responding, and locomotor pointing (Laurent \& Cavallo, 1985; Loomis et al., 1992; Rieser, Ashmead, Talor, \& Youngquist, 1990; Thomson, 1983). A generalization of this method for use with nonvisual modalities requires a neutral term; accordingly, we have opted for perceptually directed action. In perceptually directed action, the observer is presented with sensory information about the location of a target; typically, the observer receives this information from a fixed target location. Then, when the observer is ready, further access to sensory information about the target is eliminated (e.g., by blindfolding, in the case of vision), and the observer attempts to indicate his/her knowledge of the target location by one of several spatial actions. The most common action used in research of this kind is perceptually directed walking, where the observer attempts to walk to the location of the target, as it was perceived from the exposure location (Ashmead et al., 1995; Corlett, 1986; Loomis et al., 1992; Rieser et al., 1990; Speigle \& Loomis, 1993; Thomson, 1983). Loomis et al. (1992) and Fukusima, Loomis, and Da Silva (1997) have employed two actions based on the principle of triangulation to assess visually perceived distance. In triangulation by pointing, the observer views the target and then attempts to point at the target with the arm while walking blindly past it along an oblique path. In triangulation by walking, the observer views the target and then begins walking along an oblique path without vision; on command, the observer then turns and faces the target or walks some distance in its direction. In the former case, the initial and terminal pointing directions are used to "triangulate" the initially perceived target location; similarly, in the latter case, the terminal heading (facing direction) or terminal course (direction of travel) of the observer after the turn is used, along with the initial target direction, to triangulate the initially perceived target location. A virtue of methods based on perceptually directed action is their face validity: These spatial behaviors are intimately tied to space perception, they are completely natural for observers to perform, and they require no training.

All perceptually directed action tasks involve several distinct processes (Böök \& Gärling, 1981; Loomis et al., 1992; Rieser \& Rider, 1991). The first of these is perceiving the target location on the basis of sensory information received during stimulus presentation. The second involves sensing of self-motion -whether pure rotation, pure translation, or a combination of the twoand integration of sensed self-motion to obtain current position with respect to the origin of locomotion; sens- ing of self-motion is based on information about velocity and acceleration, either sensed information or commands to the musculature. The entire second stage of processing is referred to as path integration or dead reckoning (Fujita, Loomis, Klatzky, \& Golledge, 1990; Gallistel, 1990; Israel \& Berthoz, 1992; Klatzky, Beall, Loomis, Golledge, \& Philbeck, 1998; Loomis et al., 1993; Maurer \& Seguinot, 1995; Potegal, 1982). The third internal process is spatial (or imaginal) updating of the initially perceived target according to the estimate of the person's current location; spatial updating has been the focus of considerable research (e.g., Amorim, Glasauer, Corpinot, \& Berthoz, 1997; Böök \& Gärling, 1981; Loarer \& Savoyant, 1991; Pick \& Rieser, 1982; Potegal, 1971, 1972; Presson \& Montello, 1994; Rieser, 1989; Rieser, Guth, \& Hill, 1986; Rieser \& Rider, 1991).

Systematic error within any of the processes underlying perceptually directed action ought to manifest itself as systematic error in the observer's behavior, unless the systematic errors of the different processes cancel one another, which is an unlikely possibility. The nonsystematic errors (noise) associated with the different processes ought to combine additively in determining the behavioral error (assuming uncorrelated noise).

A method similar to the triangulation methods mentioned above is that developed by Böök and Gärling (1981) for the purpose of investigating properties of maintenance of orientation, their inclusive term for the two processes of path integration and spatial updating. Like the other triangulation methods, their method involved the observer's viewing a target, then walking along a straight path without further access to the target, and then indicating the direction to the previously viewed target. However, instead of using the pointing direction of the arm or the observer's heading or course following a turn toward the target after the blind traverse, their method made use of the observer's verbal report of target azimuth from the terminal point. Given that their interest was with maintenance of orientation, they obtained the observer's verbal judgments of target distance from the observation and terminal locations and used the former judgments in an attempt to partial out errors in the perception of target location from the observation point.

Still another method for measuring perceived target location is a variant of directed walking and triangulation by walking, one used by Philbeck, Loomis, and Beall (1997) in a study of visually perceived distance. In this study, observers viewed a target from a fixed location and then, when prepared to respond, donned a blindfold. They were then instructed to walk blindly to the target either along a direct path or along one of two indirect paths; the latter were initially oriented obliquely to the direct path and differed in the length of the initial segment prior to the turn toward the target. For a given target (at 1.5, 3.1, or $6.0 \mathrm{~m}$ away from the origin), a given observer tended to come to a stop at about the same location independently of the path taken. Moreover, this common terminal point was closer to the physical target location when visual dis- 
tance cues were abundant (in a fully lighted room) than when distance cues were restricted (illuminated target in an otherwise dark room). These results support three conclusions: (1) Observers were directing their action toward the initially perceived and updated target location, (2) the perceived target location depended on the availability of egocentric distance cues, and (3) path integration and spatial updating were performed without systematic error in all conditions.

The primary concern of the present study was with the accuracy of auditory distance perception of stationary sources heard in natural environments as measured using verbal reports and perceptually directed action; for purposes of comparison, we used the same measurement methods to assess the localization of visual targets presented in the same environments. Experiment 1 compared the verbal reports and perceptually directed walking of gymnasts and nongymnasts in response to visual and auditory targets. Experiments 2 and 3 used the experimental methods of Böök and Gärling (1981) and of Philbeck et al. (1997) to assess the consistency of the estimates of target location from two different "vantage points" (the point of observation and the terminal point following a short walk along a straight path).

\section{EXPERIMENT 1}

In Experiment 1, we compared verbal report and perceptually directed walking as measures of perceived distance in the auditory and visual modalities. In addition, we compared two populations of observers who might be expected to perform differently on perceptually directed tasks: gymnasts and nongymnasts. Gymnasts were chosen as one of the populations because their action is presumably well calibrated with respect to their perception.

\section{Method}

Observers. The observers were 10 members of the gymnastics team of the University of California, Santa Barbara, and 10 other students who were not members. The latter had varying degrees of interest in athletics; 2 were members of other teams (track and soccer).

Procedure. The experiment took place in a large flat grassy field with no obstructions; the nearest building was at least $70 \mathrm{~m}$ away. The observer either viewed a target at some location or heard sound emanating from that location. The auditory stimulus was recorded speech of a synthesized male voice; the synthesized speech was generated by a speech synthesizer (RC Systems Model VH600/1 operating in text-to-speech mode using default parameters). Repeating speech signals were recorded on audiotape and later played back in the experiment on a Realistic Model CTR-66 cassette recorder (with a loudspeaker $5 \mathrm{~cm}$ in diameter). The recorded speech string ("speaker one") was repeated three times during playback, for a total duration of about $5 \mathrm{sec}$. The cassette recorder was positioned on a tripod at approximately ear level and oriented upward, so that the sound radiated uniformly in all directions within the horizontal plane of the speaker. The speech sound level was $70 \mathrm{~dB}(\mathrm{~A})$, as measured from $1 \mathrm{~m}$ away using a General Radio 1551-C soundlevel meter. Because there were no nearby buildings or trees, what little reverberation was present came from the ground surface.
A visual target consisted of the cassette recorder and the tripod supporting it. The observer was instructed to close his/her eyes after approximately $5 \mathrm{sec}$ of viewing.

For both vision and auditory conditions of the experiment, the observers donned a hearing protector (Willson CP-365) between trials and before beginning to walk on the trials involving walking. The hearing protector had a relatively flat attenuation of about $38 \mathrm{~dB}$ from 1000 to $8000 \mathrm{~Hz}$ and a falloff of attenuation below $1000 \mathrm{~Hz}$; the noise reduction rating was 25 .

There were three experimental variables: target modality (auditory or visual), target distance $(4,8,12,16 \mathrm{~m})$, and response modality (walking or verbal report). The response was made by walking to the presented location or verbally reporting the distance in feet or yards. On walking trials, the target tripod was silently removed from its location while the observer walked toward it. As in all of the experiments reported in this paper, the observer never received feedback about the accuracy of his/her performance. To avoid any feedback on walking trials, the observer was prevented from seeing the target location after completing the response and was led, with eyes closed, back to the observation point.

Target modality was manipulated in blocks, with half the observers starting with the auditory modality and half the visual modality. In each block, all combinations of target distance and response modality were presented in random order, with two replications per condition. Only one response was made per trial, and the observer was not informed of the required response until after stimulus exposure.

Twelve dummy trials were interspersed with the experimental trials so that the observers would not realize that there was a restricted set of target distances. These used six distances, ranging from 3 to $18 \mathrm{~m}$, for each response type (walking and verbal). Fourteen practice trials without feedback were provided before each block, using seven distances ranging from 3 to $18 \mathrm{~m}$ for each response type.

We used a procedure that allowed the observers some familiarity with source intensity. Before the experiment, the observers stood next to the cassette recorder with their eyes closed and reached out to touch the recorder while the speech signal was played. This procedure allowed the observers to develop a remembered standard for the subsequent trials in which the source was heard from much larger distances.

\section{Results and Discussion}

The data were averaged over replications. A preliminary analysis indicated that there was a clear carryover across blocks, such that responses to visual targets were affected by experience with auditory targets, and vice versa. This resulted in the auditory and visual responses being forced closer together in the second block of trials. The nature of the order effect prompted us to concentrate on the first block only, thus treating target modality as a between-observer variable.

The data for the first block are shown as the lowermost functions of Figure 1 (verbal responses) and Figure 2 (walking responses), shown separately for gymnasts and nongymnasts. The data for the nongymnasts have been displaced vertically for purposes of clarity; the associated thin straight lines represent perfect performance. Error bars represent 1 standard error of the mean (SEM) and reflect pure between-observer variability. Because the verbal responses manifest much greater variability, we analyzed the walking and verbal responses separately. 


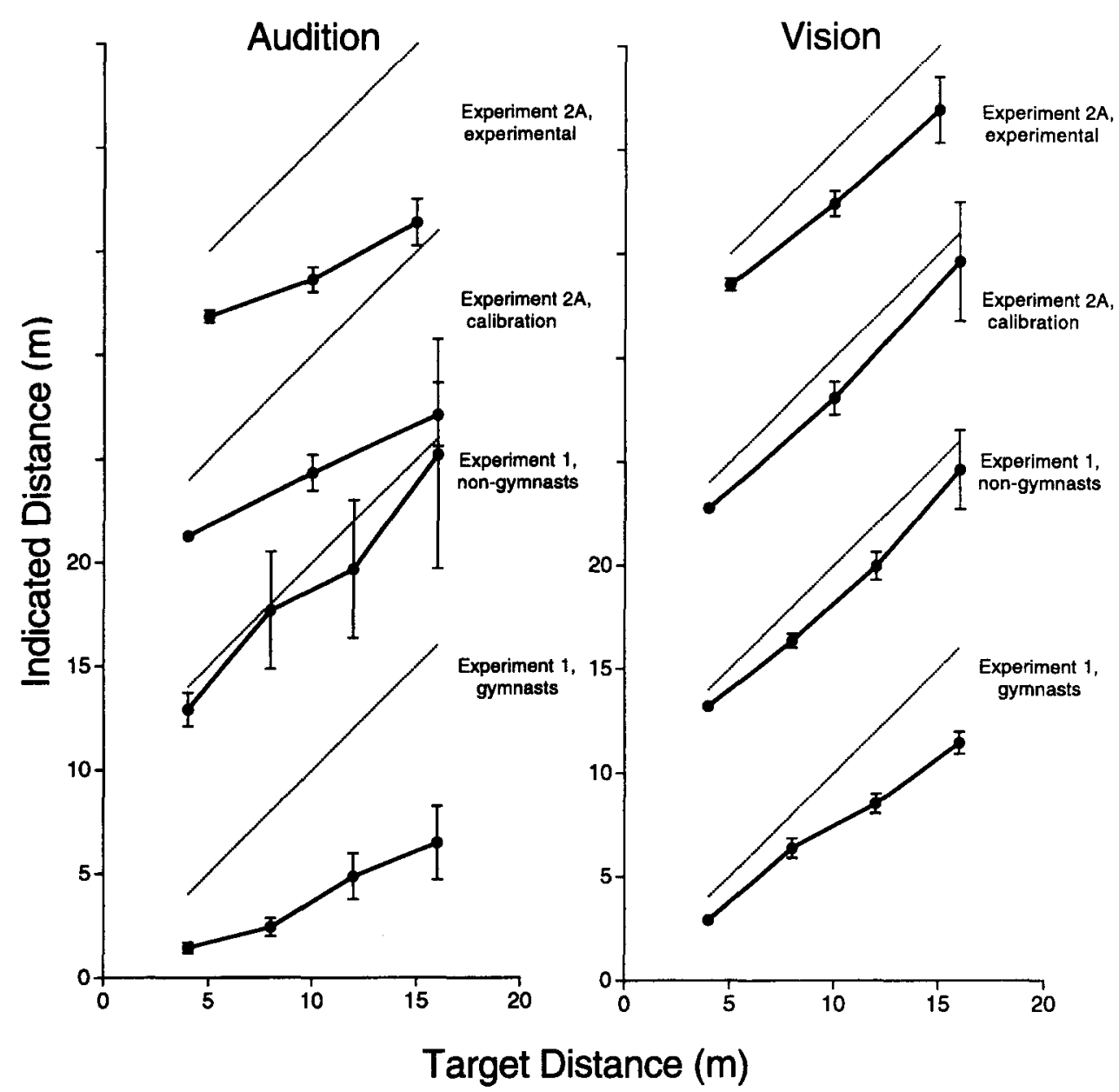

Figure 1. Results of the three experiments obtained with verbal responses. Except for the gymnasts' data of Experiment 1, the data functions have been displaced vertically for purposes of clarity; the associated thin straight lines represent perfect performance. The error bars represent $1 S E M$.

The analysis of variance (ANOVA) on walking responses showed the expected effects of distance $[F(3,48)=$ $130.0, p<.0001]$ and target modality $[F(1,16)=17.06$, $p<.001]$, as well as an interaction between them $[F(3,48)$ $=11.54, p<.0001]$. There were no other effects. The ANOVA on verbal reports showed an effect of distance $[F(3,48)=40.87, p<.0001]$ and an interaction between distance and athletic status $[F(3,48)=2.90, p<.05]$.

As mentioned, between-observer variability was generally greater for verbal report than for walking. In a similar comparison, Philbeck and Loomis (1997) found smaller between-observer variability with the motoric response in one experiment but comparable values in another. Also, Philbeck and Loomis found that the verbal report and walked distance measures were tightly coupled across viewing conditions; they concluded that the two measures were controlled by the same internal variable (perceived distance). The data for the gymnasts in Figures 1 and 2 are consistent with this: The mapping that relates the verbal responses to those for walking in the visual condition is about the same as that for the auditory condition. For example, when the mean verbal response is about $6 \mathrm{~m}$, the mean walking response is about $8 \mathrm{~m}$, regardless of target modality. The large variability for the verbal responses of the nongymnasts precludes drawing any clearcut conclusion on this same issue.

Focusing on the results for walking, the present experiment showed that the gymnasts, as a group, performed about the same as the nongymnasts, for both vision and audition. For vision, the two populations showed little systematic error in walking to targets-a result that has been obtained in many other studies. In contrast, both observer populations showed an approximately twofold compression of walked distance relative to auditory target distance, with underestimation at all but the nearest distance. On the basis of the more reliable walking data, the conclusion appears inescapable that, at least under the conditions of this experiment, perceived auditory distance varied considerably less than perceived visual distance for the same range of target distances. 


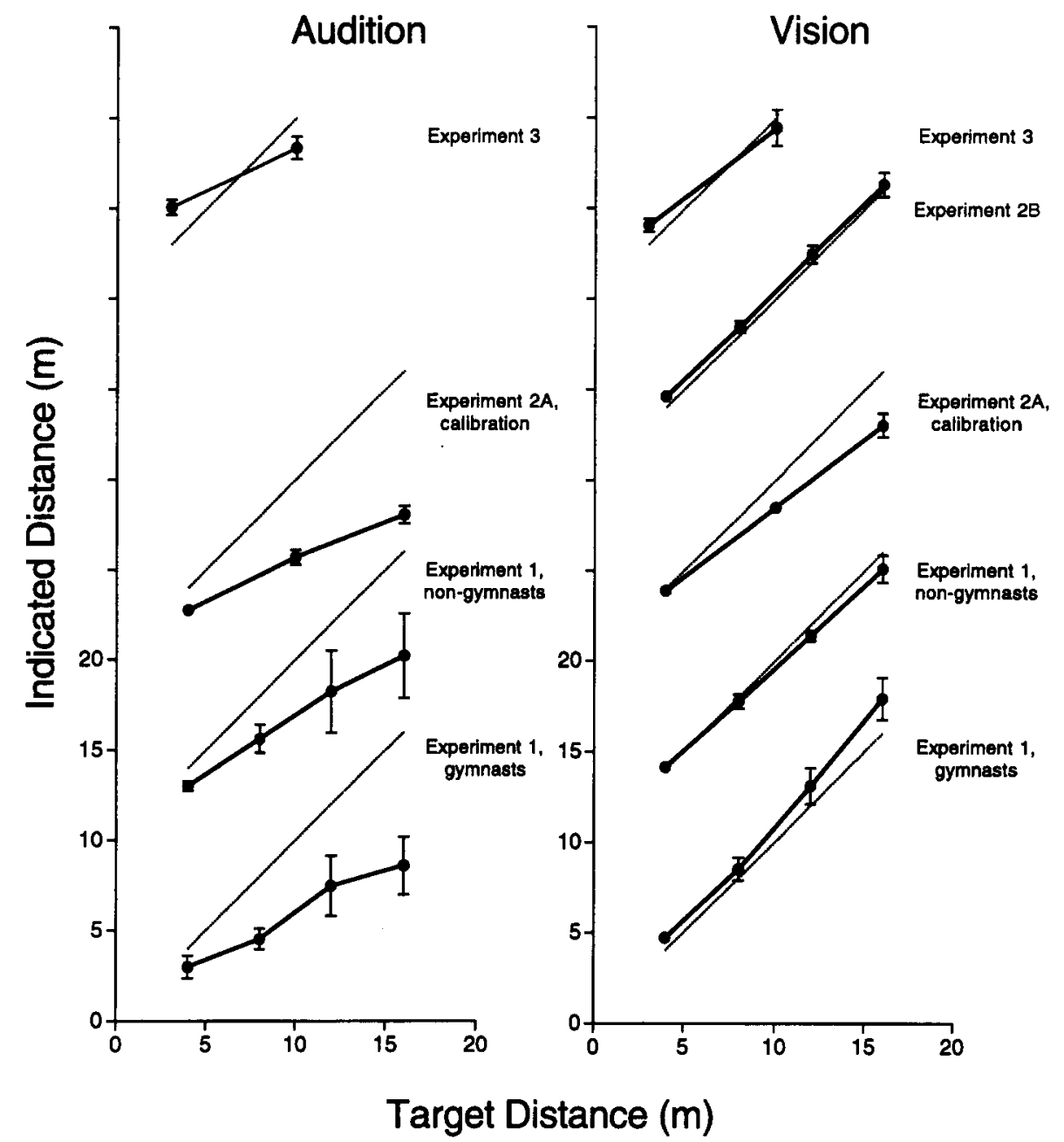

Figure 2. Results of the three experiments obtained with walking responses. Except for the gymnasts' data of Experiment 1, the data functions have been displaced vertically for purposes of clarity; the associated thin straight lines represent perfect performance. The error bars represent $1 S E M$.

\section{EXPERIMENT 2A}

Experiment $2 \mathrm{~A}$ had two goals. The first was to use the method of Böök and Gärling (1981) to assess the consistency of verbal estimates of target location from two different points in space: the point of observation and the terminus of a short walk along a straight path. Observers verbally reported the distance and azimuth of targets (visual or auditory) either from the exposure location (origin) or from a location $5 \mathrm{~m}$ distant from the exposure location (the terminus of a walk during which the observer received no additional perceptual information about the target location). In the walking condition, we compared performance following exposure to one or two targets to determine whether there is greater systematic error associated with the spatial updating of two targets than there is with one. Target modality was varied between observers. Number of targets and point of response varied within observers. The trials associated with this task are referred to as experimental trials.
The second goal, like that of Experiment 1, was to assess the accuracy of verbal reports and motoric responses and to compare these two types of responses. We intended to use the data from these calibration trials to create calibration functions for both vision and audition that would permit the mapping of the verbal reports of azimuth and distance from the experimental trials into the corresponding values for walking. The observers made verbal reports of target azimuth and distance from a fixed observation point. For the motoric responses to distance, the observers were exposed to the target from the same observation point and then attempted to walk to its location without receiving additional stimulation from the target during the traverse. For motoric responses to azimuth, the observers were exposed to targets varying in azimuth from the same observation point; the observers then attempted, without further stimulation, to turn and face the initial direction of the target. In connection with our comparison of verbal and motoric measures of azimuthal responses to sounds, we note the extensive study 
by Haber, Haber, Penningroth, Novak, and Radgowski (1993) comparing nine methods of indicating the direction of a sound source. In their study, however, observers completed the directional response (including head turning) before the sound was turned off; in our experiment, the stimulus exposure was terminated before the response was initiated.

\section{Method}

Observers. The observers were 14 students at the University of California, Santa Barbara, who were paid for participating. They were divided into two groups, half receiving auditory targets and half visual targets. The duration of the experiment was approximately $1.5 \mathrm{~h}$.

Procedure. The experiment took place in the same field as in Experiment 1 . The visual and auditory stimuli were the same except that, in this experiment, either one or two stimuli were presented on a given trial. For auditory stimulation, two cassette recorders were used, referred to below as Speaker 1 and Speaker 2. The recorded speech string ("speaker one" or "speaker two") was repeated three times during playback, for a total duration of about $5 \mathrm{sec}$ for each speaker. The observers were permitted to rotate their heads during the observation. On vision trials, the target consisted of the cassette recorder and the tripod supporting it; when two targets were used, they were distinguished by visible labels ("1" and "2") attached to each. The observer was instructed to close the eyes after approximately $5 \mathrm{sec}$ of viewing when only one target was present and after $10 \mathrm{sec}$ of viewing when two were present. As before, for both vision and auditory conditions of the experiment, the observers donned the hearing protector between trials and before beginning to walk on those trials involving walking.

There were three types of trials: experimental trials, calibration trials, and dummy trials. These were randomly intermixed. In the experimental trials, the observer verbally reported the target's distance (in feet), azimuth (in degrees), and side (right or left), usually in that order. The targets were at combinations of three distances $(5,10$, and $15 \mathrm{~m})$ and six azimuths $\left(-25^{\circ}, 25^{\circ},-70^{\circ}, 70^{\circ}\right.$,

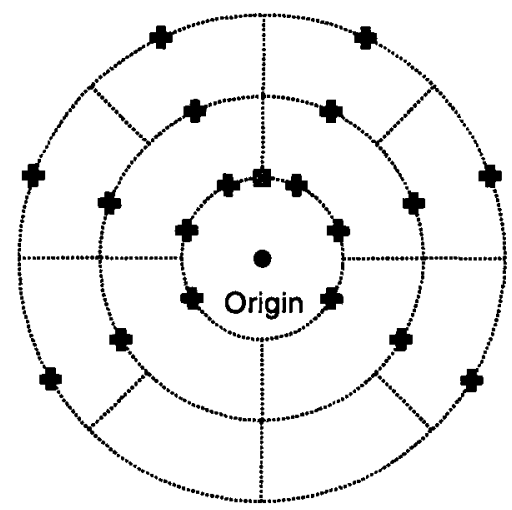

$+\quad$ Target Locations

- Terminus (for walking trials)

Figure 3. Target locations used for the experimental trials of Experiment $2 \mathrm{~A}$. The concentric circles represent radii of 5,10 , and $15 \mathrm{~m}$. Observers viewed or listened to the targets from the origin. On nowalking trials, they made their verbal reports from the origin. On walking trials, they walked with occluded vision and hearing to the terminus on the 5-m circle and made their verbal reports from there. $-120^{\circ}$, and $120^{\circ}$ ), constituting 18 experimental locations (see Figure 3). Zero azimuth is the reference direction for the experimental workspace in which all stimuli were arranged; it coincided with the observer's heading (facing direction) on all trials and with the observer's course (travel direction) on all walking trials. Positive azimuth values represent angles to the right; negative values represent angles to the left.

The experimental trials used either one target or two targets; however, on two-target trials, the observer responded to only one of these (the probe) after its designation by the experimenter following the observation interval. In the one-target auditory condition, Speaker 1 was used on half the trials, and Speaker 2 was used on half the trials. In the two-target auditory condition, the two speech signals (corresponding to Speakers 1 and 2) were presented in succession, each lasting $5 \mathrm{sec}$ and separated by $1 \mathrm{sec}$. Speaker 1 was presented first on half of these trials and second on the other half of these trials. In the one-target visual condition, the observer was allowed to view the target for $5 \mathrm{sec}$; whereas, in the two-target visual condition, the observer was allowed $10 \mathrm{sec}$, to view both targets as he or she wished. In both visual and auditory conditions, the two targets were always on opposite sides of the observer's median plane; in addition, the two targets never shared the same distance or absolute azimuth (e.g., $-25^{\circ}$ and $25^{\circ}$ ). Each target served as the probe half the time; the observer was informed of which target was the probe using the identifying label just before the observer made his/her verbal response.

The third variable on experimental trials was whether the observer responded from the original exposure location (origin) or from the terminus of a short straight walk; the variable is referred to hereafter as point of response. In the walk condition, the observer was signaled to walk forward before being informed of the probe. The observer donned the hearing protector; after the observer had walked $5 \mathrm{~m}$, the experimenter tapped the observer on the shoulder, indicating that he/she should stop. The observer removed the hearing protector, was informed of which target was the probe, and then made his/her response. In the respond-at-origin condition, the observer was signaled to report immediately after the observation interval. No delay was imposed to match the duration of walking because it seemed unlikely to have any effect, given that the observers could easily rehearse the to-be-reported locations.

The manipulation of point of response and number of targets occurred over three conditions: respond-at-origin, two targets; respond-at-terminus, one target; respond-at-terminus, two targets. Implementing these conditions at each of the 18 locations constituted a total of 54 experimental trials. The trial order was completely randomized for each observer.

So that the observer could not anticipate the response that would be required after walking, while initially encoding the target (particularly a concern with visual exposure), we introduced dummy trials in which the observers walked approximately $3 \mathrm{~m}$ before responding. Target locations on these trials were drawn from those used on experimental trials, and the dummy trials were interspersed among them. There were 10 dummy trials: 5 with one target and 5 with two targets.

Intermixed with experimental and dummy trials were 24 calibration trials in which the observer estimated either the distance or the azimuth of a single target with a verbal or motoric response. The motoric response was directed walking to the target (distance calibration) or turning toward it (azimuth calibration). Walking responses were measured with a tape measure. Turn responses were measured from angles marked on a response board on which the observer stood. A central post and heel bar allowed the observers to orient themselves while the targets were presented; the heel bar was removed to allow free turning before the response was made. The response modality was not known in advance. The distance calibration trials used locations at zero azimuth and distances of 4 . 
10 , and $16 \mathrm{~m}$; the azimuth calibration trials used locations at a distance of $9 \mathrm{~m}$ and azimuths of $20^{\circ}, 65^{\circ}$, and $125^{\circ}$. For each response (verbal or motoric), there were two replications of distance calibration and one of azimuth calibration on each side.

Before beginning the experiment proper, the observers received one practice trial in each condition (four calibration types, defined by reported parameter and response modality, and three experimental types [origin/2-targets, terminus/1-target, terminus/2targets]). Unlike in Experiment 1, the observers here did not get to touch the cassette recorder while speech was being played; consequently, in Experiment 2 (which was first in chronological order), the observers were not given explicit information allowing them to relate speaker loudness to its egocentric distance.

\section{Results}

Analysis of azimuth. When azimuth was judged from the observation point, the mean signed error (averaged over observers) for different target azimuths was usually less than $5^{\circ}$ on calibration and experimental trials for both vision and audition and for both types of responding (verbal and motoric). This high accuracy of the average azimuthal judgments is not unexpected, especially since the observers were permitted to make head rotations during the observation. Because the focus in this article is on distance perception, we omit all analyses of the azimuth judgments except as they are needed for comparing the judged locations of the target location before and after walking on the experimental trials.

Calibration trials. The indicated distance responses are shown in Figure 1 (verbal) and Figure 2 (walking) as a function of target distance. They have been displaced vertically for purposes of clarity; the associated thin straight lines represent perfect performance. The error bars represent $1 S E M$ of between-observer variability. The distance trials showed that, regardless of response modality, responses increased with physical distance $[F(2,24)=$ $66.31, p<.001]$. More importantly, there was a main effect of target modality $[F(1,12)=18.31, p<.001]$ and a modality $\times$ distance interaction $[F(2,24)=6.33, p<$ $.01]$. Observers' responses to auditory targets showed a greater compression of indicated distance relative to target distance, yielding slopes of less than .5 for the function relating indicated distance to the actual value. With visual targets, these slopes were much closer to 1.0 , but there was some underestimation of distance, especially for walking to the far distances. By and large, the walking data for distance are concordant with those of Experiment 1 .

The ANOVA on the calibration data included response modality as a variable. Because there were no main effects or interactions involving response modality, we conclude that the verbal reports in Experiment 2 did not differ significantly from the walking responses. For this reason, we did not construct calibration functions for mapping the verbal reports of the experimental trials into walking responses, as we had intended. We take the near congruence of the two response measures to mean that the verbal reports obtained in the experimental trials are valid indicators of perceived (and updated) distance.
Experimental trials. ANOVAs on the experimental trials deleted responses that were more than 6 standard deviations above or below the mean. Rejection of either azimuth or distance data led to rejection of the entire trial. Ultimately, 12 responses were eliminated. The remaining data were averaged over targets on the left and the right side.

Figure 4 shows the actual location of each target and the centroid of reported locations (averaging over observers) by target modality and the three experimental conditions. For the origin condition, the reported azimuth and distance values constituted an estimate of the target location with respect to the observation point. For the two terminus conditions, the reported azimuth and distance values constituted an estimate of the target location with respect to the terminus of the $5-\mathrm{m}$ walk. To the extent that the observers correctly perceived the $5-\mathrm{m}$ walk and correctly updated the initially perceived target location, the estimated target locations (with respect to the origin) for a given target should be the same in all three conditions.

One clear result seen in Figure 4 is that the estimated target locations were much closer to the physical target locations for vision than for audition. Another clear result is that the estimates of target location (with reference to the observation point) systematically differed between the origin and terminus points of response. For both modalities, targets estimated as closer than $5 \mathrm{~m}$ in the origin condition tended to be estimated as more distant (relative to the point of observation) in the two terminus conditions. In addition, the estimated target locations differed in direction (re the observation point) between the origin and terminus conditions for audition. Finally, there was no obvious effect of number of targets on the reported target locations, as judged from the termimus point of response.

The mean verbal reports of distance from the observation point, averaging over observer and azimuth, are plotted in Figure 1, next to the label "Experiment 2A, experimental." Error bars represent between-observer variability (1 SEM).

The ANOVA on these verbal distance estimates showed an effect of target modality $[F(1,6)=32.95, p<.001]$, an effect of distance $[F(2,12)=44.34, p<.0001]$, and an interaction between target modality and distance $[F(2,12)=13.19, p<.01]$. This effect reflects the fact that indicated distance for audition was more compressed than indicated distance for vision.

\section{Discussion}

The calibration experiment produced three findings: (1) Verbal and walking responses to distance were largely concordant, (2) indicated distance in the auditory condition varied much less than indicated distance in the visual condition, replicating the primary result of Experiment 1 , and (3) there was some undershooting of the walking responses to visual targets at the two longer dis- 


\section{Audition Vision}
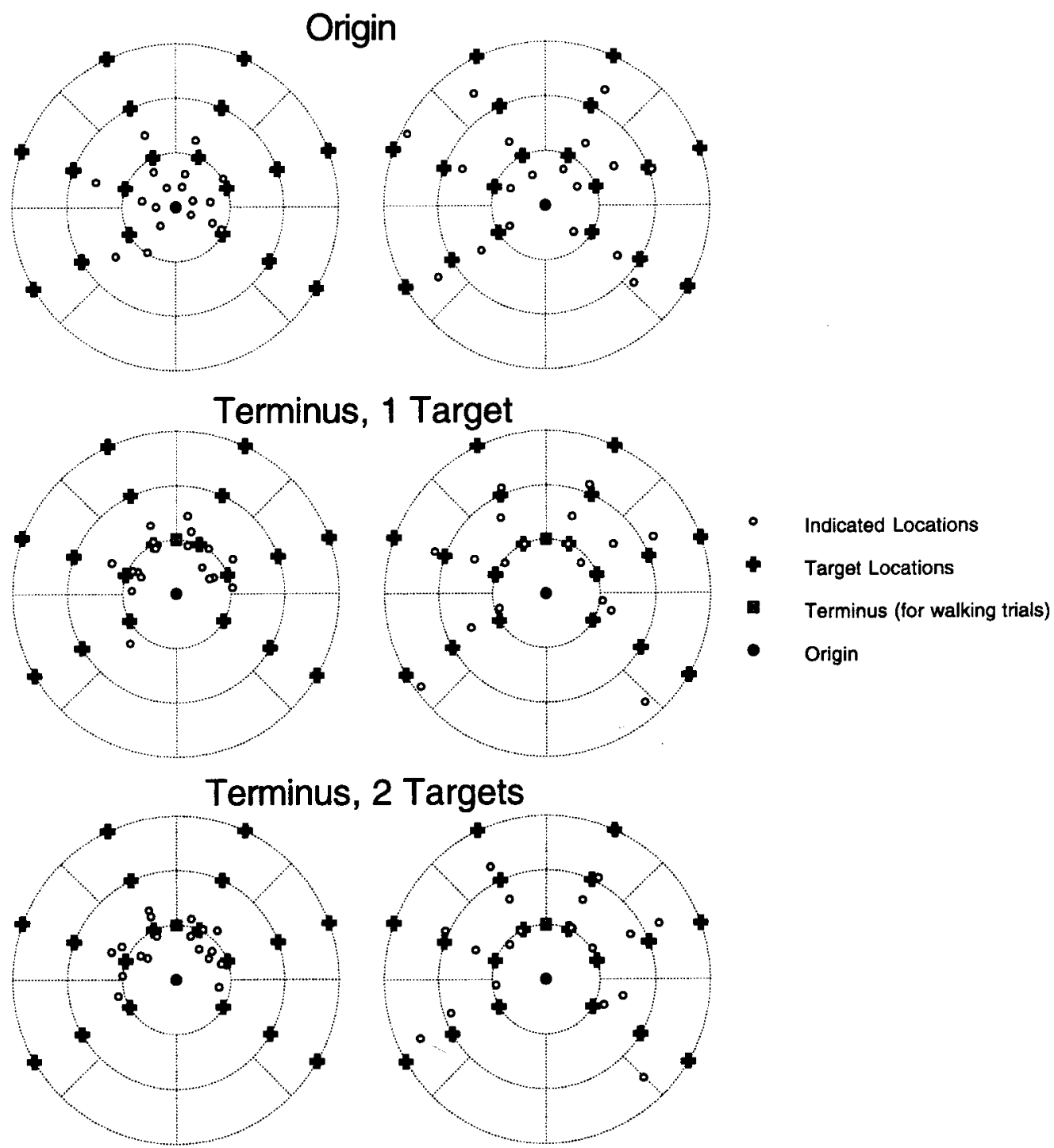

Figure 4. Results of the experimental trials of Experiment 4. Target locations are represented by the solid crosses. For each, there is a corresponding open symbol that represents the centroid of estimated locations made by different observers. In the no-walking condition, observers viewed or listened to two targets from the origin and then were told which target location to estimate. The verbally reported azimuth and distance determined the estimated location. In the walking conditions, the observers viewed or listened to one or two targets. Then, without vision and hearing, they walked to the terminus on the 5 -m circle. After the walk, the observers verbally reported the azimuth and distance of the spatially updated location of the target.

tances. The main experiment employing verbal report also yielded three primary findings: (1) Auditory targets were estimated as closer than visual targets at the same distance, (2) the estimated target locations were not the same in the origin and terminus conditions, and (3) there was no effect of number of targets on the systematic errors associated with their localization. This latter result supplements the finding of Rieser and Rider (1991) that varying the number of visual targets from one to five in a spatial updating task had no effect on either constant (systematic) errors or variable (corresponding to precision) errors either for adults or for 4-year-old children.
If the observers were correctly sensing their self-motion during walking, were correctly updating the locations of the initially perceived locations, and were correctly reporting these updated locations, the estimated target locations should have been the same in the respond-atorigin and respond-at-terminus conditions. This is true even if the perceived locations were quite discrepant with respect to the physical target locations (see Philbeck et al., 1997).

If the observers were overestimating or underestimating perceived self-motion during the walk by $X \mathrm{~m}$, this should have caused the estimated target locations in the 
terminus conditions to be displaced from the estimated target locations in the origin location by $X \mathrm{~m}$ in a direction parallel to the walking direction (again, this is true whether or not the perceived target locations were accurate). A uniform displacement of estimated target locations from the top panel of Figure 4 (Origin) to those in the two lower panels (Terminus) was not the result obtained; consequently, we conclude that systematic error above and beyond misperception of target location and misperception of self-motion must be involved. We believe that such an error is somehow being introduced by the procedure of using verbal report to assess spatial updating. The results of the experiment by Philbeck et al. (1997) and Experiment 3 below suggest that perception of self-motion and spatial updating are performed without systematic error, lending support to the suggestion that additional systematic error is associated with verbal report in a spatial updating task.

\section{EXPERIMENT 2B}

For the group averages in Experiment 2A, we found a clear underresponding to the more distant visual targets in Experiment 2A; this was true both for the verbal estimates in the main experiment and for the walking responses in the calibration experiment. This systematic underestimation conflicts with the general finding that perceptually directed walking to visual targets under fullcue viewing exhibits little systematic error. Accordingly, we conducted a control experiment, this time in a smaller field. The observers viewed a target that varied in its egocentric distance (from 4 to $16 \mathrm{~m}$ ) and then attempted to walk to it without vision. In three target conditions, the observers fixated (1) the top of a tripod, which was at eye level, (2) the base of the tripod, which was at ground level, or (3) a white plastic ball placed on the ground. There was no effect whatsoever of this manipulation.

The mean data, averaged over observers and the three target conditions, are given in Figure 2; they confirm the general finding that blind walking to previewed targets under full-cue conditions is without systematic error at least up to $15 \mathrm{~m}$.

\section{EXPERIMENT 3}

In Experiment 2A, it was found that the estimated target locations in the origin and terminus conditions were sometimes discrepant, especially for audition. We interpret this as the result of additional errors introduced in the verbal estimation process in tasks involving spatial updating. Experiment 3 was much like Experiment 2A, except that motoric responses rather than verbal responses were used. Experiment 3 was very similar to that of Philbeck et al. (1997), except that they measured the entire walking trajectories and we measured only the terminal locations of the observers' walks. The basic idea was that an observer, after initially viewing or hearing a distant target, ought to walk to the same location in space (its per-
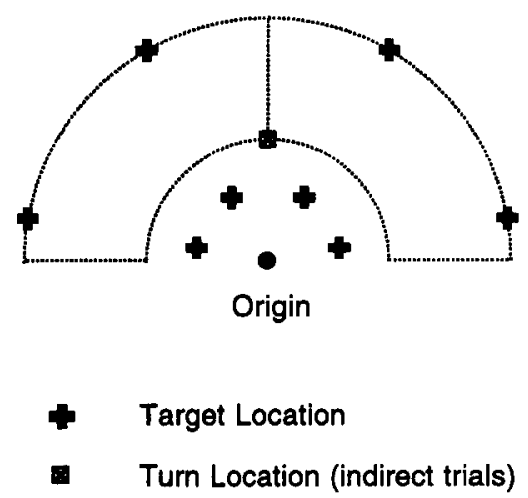

Figure 5. Target locations used for the experimental trials of Experiment 3. The concentric circles represent radii of 5 and $10 \mathrm{~m}$. Observers viewed or listened to the targets from the origin. On some trials, observers then attempted to walk directly to the target without further perceptual information about the target location. On other trials, they attempted to walk to the target along an indirect path. For these trials, they were led by an experimenter to a location at $0^{\circ}$ azimuth on the 5-m circle and then attempted to walk the rest of the way on their own.

ceived location) whether walking directly toward it or along an indirect path with a turn point specified by the experimenter. This perceived location need not coincide with the physical location of the target.

\section{Method}

Observers. The observers were 14 students at the University of California, Santa Barbara, who were paid for their participation. Seven observers performed in the vision conditions, and 7 performed in the audition conditions.

Procedure. The design was similar to that of Experiment 2A. Target modality was manipulated between observers. Also varied were number of targets (one or two) and type of response (direct or indirect walking). These variables were crossed. In each of the four resulting conditions, the observers took part in eight experimental trials, corresponding to targets at combinations of two possible distances ( 3 or $10 \mathrm{~m}$ ) and four azimuths $\left(-30^{\circ}, 30^{\circ},-80^{\circ}\right.$, and $80^{\circ}$ ). The configuration of target locations is shown in Figure 5. On trials that presented two stimuli, the second was in an experimental location that did not duplicate the absolute azimuth or distance of the target and was on the opposite side of the first.

Within each condition, there were also five dummy trials intended to discourage the observers from trying to plan the required response before walking. On dummy trials involving walking forward before responding, the observer was stopped either before or after the 5-m distance used on experimental trials. The locations on dummy trials were at distances of 3,7 , or $10 \mathrm{~m}$ and azimuths of $30^{\circ}, 55^{\circ}$, or $80^{\circ}$, each side occurred equally often.

As in Experiment 1, we used the procedure that allowed the observers some familiarity with source intensity. Before the experiment, the observers stood next to the cassette recorder with their eyes closed and reached out to touch the recorder while the speech signal was played.

\section{Results}

Figure 6 shows the terminal points of all 7 observers (in the vision conditions with one target) who attempted to walk either directly (left panel) or indirectly (right panel) to the target located $10 \mathrm{~m}$ at $30^{\circ}$ right azimuth. The 


\section{Direct Walking, Vision}

\section{Indirect Walking, Vision}
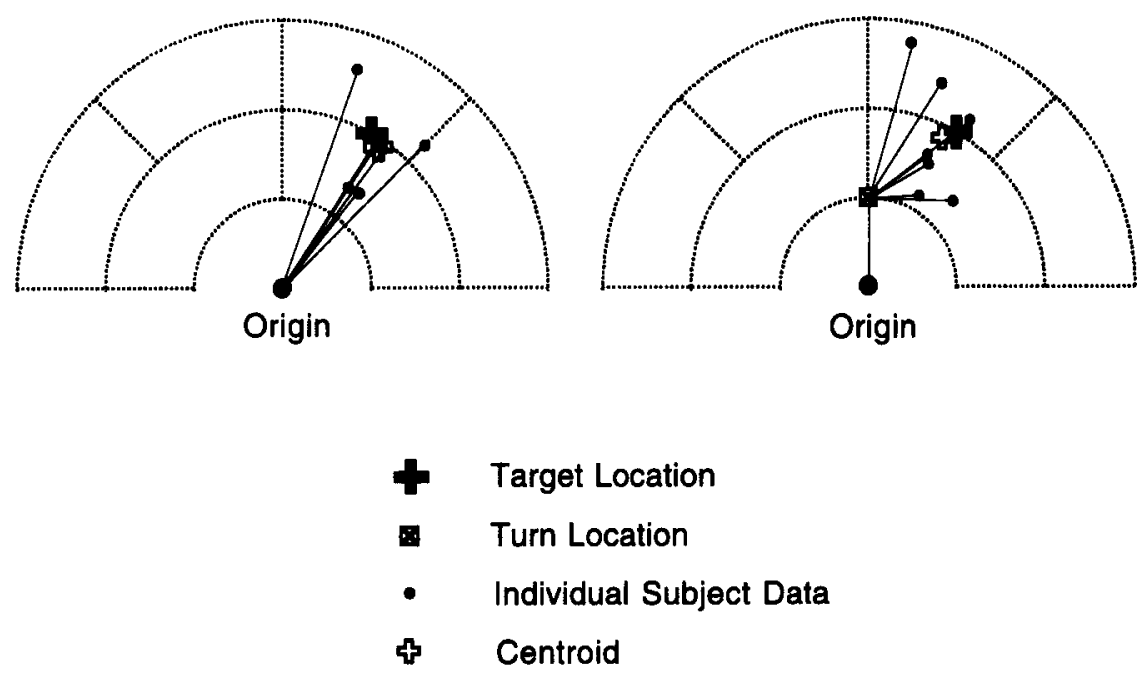

Figure 6. Individual data for one target in Experiment 3. The concentric circles represent radii of 5,10 , and $15 \mathrm{~m}$. The location of the visual target (located at $30^{\circ}$ azimuth and $10 \mathrm{~m}$ from the observation point) is indicated by the solid cross. Stopping points for the 7 observers in the direct and indirect walking conditions are shown by the solid dots. The centroid of these 7 locations is indicated by the open cross in each condition.

physical target location is indicated by the solid cross, and the centroid of the seven terminal locations is indicated by the open cross.

Figure 7 shows the centroid of the observers' stopping locations for each combination of target modality, target location, and path (direct vs. indirect). Data were pooled over number of targets (one vs. two) because there was little discernible effect of this manipulation. As can be seen from the figure, the observers as a group stopped in very nearly the same location for each target in each modality, whether they responded directly or after walking forward $5 \mathrm{~m}$. The near coincidence of the centroids
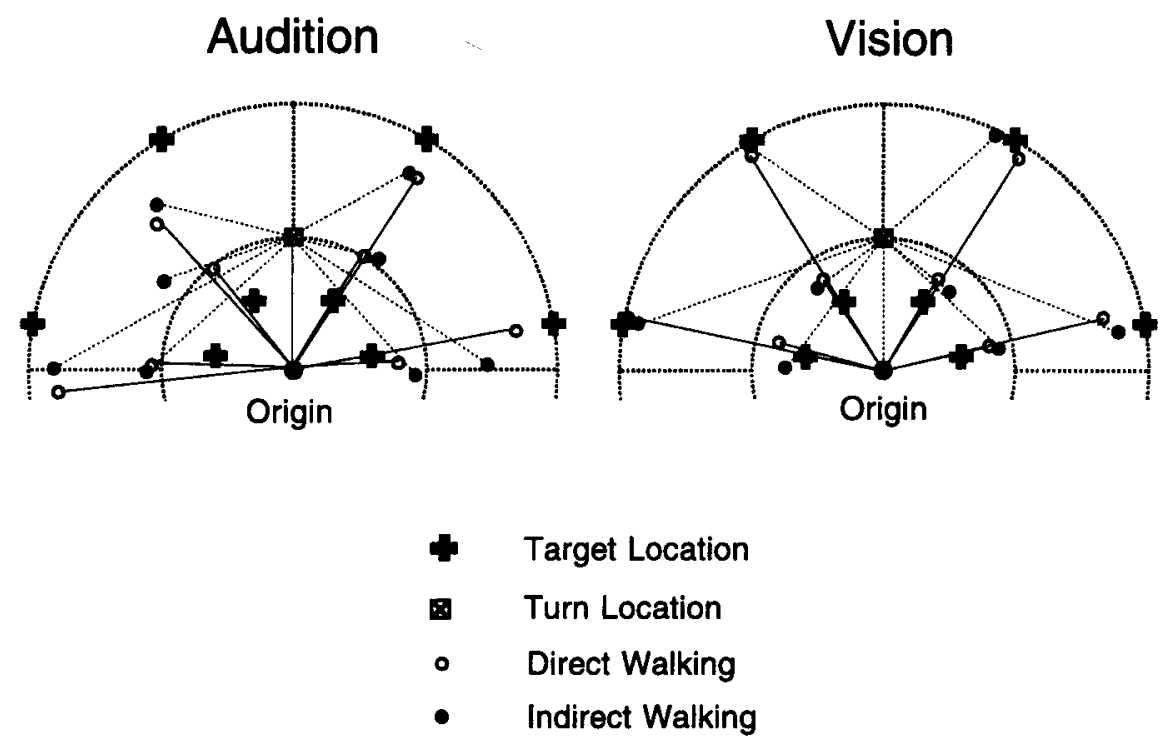

Figure 7. Results of Experiment 3. The concentric circles represent radii of 5 and $10 \mathrm{~m}$. The locations of the auditory and visual targets are indicated by the solid crosses. The centroids of the individual stopping locations (see Figure 6) are represented by the open dots for direct path and solid dots for indirect path. The lines connect each centroid with either the origin or the turn point. 
for the 3-m targets is truly remarkable: When the observers walked indirectly, they had to first walk forward $5 \mathrm{~m}$ to the turn point, turn through a large angle, and then walk back several meters to the perceived target locations.

The mean distances to the terminal point centroids, averaged over side, azimuth, path, and observer, are plotted as the uppermost functions in Figure 2. The ANOVA on distance revealed a main effect of distance $[F(1,12)=$ $121.86, p<.0001]$ and a distance $\times$ target modality interaction $[F(1,12)=7.23, p<.05]$.

To summarize, in this experiment the effect of target modality on distance judgments that was found previously was again found - namely, a greater compression of indicated distance for audition than for vision. The visual judgments were not entirely accurate, with a mean distance effect of $5.4 \mathrm{~m}$ rather than the $7 \mathrm{~m}$ corresponding to the true difference between the target distances. The distance function also differed somewhat from that in Experiment 2A, in that the observers tended to overestimate the short distances. However, the present 3-m distance was smaller than that used previously.

\section{Discussion}

In Experiment 3, the observers viewed or listened to a target from an initial vantage point and then attempted to walk along direct and indirect paths to its location without further sensory information about the target location. The results indicate that, on average, the stopping locations of the observers for a given target were nearly coincident despite the different paths taken to get there (Figure 7). The convergence of the direct and indirect paths is evidence that the observers were directing their walk to a particular location in space and that they were able to direct their walk without systematic error either in path integration (integrating perceived self-velocity) or in the spatial updating of the initially perceived target location. That it was the perceived location to which the observers were directing their walk is indicated by the dependence of the mean stopping locations on the sensory modality used while observing the target. As found in Experiments 1 and 2, the observers were more accurate in walking to visual targets than to auditory targets; here, a 7-m change in target distance (from 3 to $10 \mathrm{~m}$ ) produced a change in mean indicated distance of $5.4 \mathrm{~m}$ for vision and of only $3.0 \mathrm{~m}$ for audition. Our interpretation of this result is that auditory perception of egocentric distance exhibits more systematic error than does visual perception of egocentric distance. The results of Experiment 3 differ from the results of Experiments 1 and 2 in that the stopping locations to the near target $(3 \mathrm{~m})$ were generally more distant from the origin than the target, suggesting that the initially perceived target location was beyond the target. This tendency was true of both vision and audition, but it was stronger for audition.

\section{GENERAL DISCUSSION}

The primary concern of this paper is with the measurement of perceived egocentric distance. A number of pre- vious studies have shown that perceptually directed action (usually walking) provides relatively stable measures of perceived distance for both vision (Fukusima et al., 1997; Loomis et al., 1992; Philbeck \& Loomis, 1997; Philbeck et al., 1997; Rieser et al., 1990; Thomson, 1983) and audition (Ashmead et al., 1995; Speigle \& Loomis, 1993). Perhaps the most convincing evidence of the validity of the method comes from the study by Philbeck et al. (1997), which supports the following conclusions: (1) Observers direct their walking to the perceived location of the target, and (2) they perform the walk without systematic error either in path integration or in spatial updating of the target location. Under full-cue conditions, the average observer performed quite well; however, under reduced-cue conditions, the average observer walked beyond the two nearer targets $(1.5$ and $3.1 \mathrm{~m})$ and walked well short of the far target $(6.0 \mathrm{~m})$.

The three experiments reported here compared visual and auditory distance perception using both perceptually directed walking and verbal report. Several generalizations can be made on the basis of these experiments. First, the verbal estimates of perceived distance are more variable across groups of observers than are the estimates from perceptually directed walking, as can be seen in Figures 1 and 2 ; in particular, the gymnasts and nongymnasts were more alike in terms of walking than in terms of verbal report. Second, visual perception of egocentric distance exhibited little systematic error under the full-cue conditions of these experiments, as is apparent in the more precise walking data in Figure 2; this result is consistent with the rest of the literature. Third, auditory perception of egocentric distance exhibited considerable systematic error under the conditions of these experiments, as is especially apparent in the walking data (Figure 2) - there is a large compression of the walking responses relative to the stimulus range. Fourth, the results of Experiment 3 are concordant with those of Philbeck et al. (1997) in showing that perceptually directed walking along direct and indirect paths tends to terminate at approximately the same location regardless of path (Figure 7); this finding means that observers perform perceptually directed walking without systematic error in path integration or in spatial updating. Fifth, the results of Experiment 2 using the verbal estimation procedure of Böök and Gärling (1981) do not show the same degree of internal consistency as do those of Experiment 3; the target locations estimated from the origin generally do not coincide with those estimated from the terminus of a 5-m walk from the origin (Figure 4). A possible reason for this lower internal consistency is that, for an observer expecting to make a verbal estimate at the end of a walk, there is less engagement of attention during spatial updating than when the observer is continuously directing his/her walking with respect to the updated target. Sixth, nongymnasts as a group do not exhibit more systematic error in perceptually directed walking than skilled gymnasts (Figure 2). Seventh, there is no evidence that observers exhibit more (or less) systematic error when updating two targets than when updating one target. 
Our finding of a compression of walked distance relative to auditory target distance is consistent with the results of two other experiments using perceptually directed walking following exposure from a fixed listening location. Speigle and Loomis (1993) varied the distance of a source ( $20-\mathrm{Hz}$ pulse train) from 2 to $6 \mathrm{~m}$. The corresponding mean walked distances (for 10 observers) varied from 3.7 to $5.5 \mathrm{~m}$, a mere 1.8 -m range in comparison with the 4-m stimulus range. When the observers were allowed to walk during the stimulus exposure (providing acoustic tau and motion parallax information about source distance), the walked distances were slightly more accurate and the range of indicated distances was slightly greater. In a more thorough study, Ashmead et al. (1995) conducted four experiments with a total of $30 \mathrm{ob}-$ servers. In three of these experiments, observers participated in conditions with stationary listening of the source (white noise that had been low-pass filtered with a cutoff of $8000 \mathrm{~Hz}$ ). The experiment was conducted on a concrete sidewalk (presumably giving more reverberation than in the present experiments); sound level was made unreliable as a distance cue. For a stimulus range of 5-19 m (almost a fourfold increase), walked distance in two experiments varied from 8.9 to $17.9 \mathrm{~m}$ (a twofold increase), indicating a strong perceptual compression of stimulus range; however, unlike the results of the present experiments, the average observer overestimated target distance for most of this range. When acoustic tau information was provided by allowing observers to walk during the stimulus exposure, performance improved considerably, although there was still some perceptual compression of the stimulus range. Overall, the results of the present study and of these two previous studies using perceptually directed action indicate that perceived auditory distance is compressed in range to about half that of the stimulus range when observers listen to stationary targets.

Does the consistent finding of a compression of walked distance relative to target distance mean that auditory distance perception always exhibits systematic error? At this point, there has been too little research to give a definitive answer. Surely, the results of the present study and previous studies using walking as a response must be representative of a range of normal listening conditions. However, there may be more optimal circumstances, such as estimating auditory distance within familiar acoustic environments or within environments that are more reverberant. Also, it is possible that the observers might have performed more accurately in these experiments had the sources been located on the ground surface, providing access to an angular elevation cue, albeit a weak one; however, this manipulation would have resulted in a listening situation that is less representative, because the typical sound source is located above the ground. Another factor of possible relevance to observers' performance is experience in using sound to guide action; it is possible that skilled blind travelers, who are more accustomed to having to act on the basis of emitted and reflected sound, might be more accurately calibrated and thus might more accurately indicate the distance of sound than do sighted observers. However, the null result here in the comparison of gymnasts and nongymnasts in walking to visual targets reduces the likelihood of this possibility.

Finally, we return to the issue of distance perception with virtual acoustic displays. Although achieving externalized virtual sound with headphones has proven a major challenge, there nevertheless have been some successes (e.g., Begault \& Wenzel, 1993; Loomis et al., 1990; Wenzel et al., 1993; Wightman \& Kistler, 1989). Moreover, there is reason for optimism for achieving a considerable range of perceived distance with headphones driven by a virtual acoustic display; headphone listening is not a limiting factor per se. As Loomis and Soule (1996) have reported, the experience of very realistic sound at a considerable distance is possible with headphones, as demonstrated with a simple binaural listening device. It consists of in-ear earphones, a sound-attenuating hearing protector worn over the earphones, microphones mounted on top of the hearing protector earcups, and a stereo amplifier driving the earphones with the microphone signals. Most people listening with the device with the freedom to rotate and translate the head naturally experience the sounds as externalized and varying over a considerable range of distance (well beyond several meters). Assuming that the underlying acoustic cues (e.g., spatialized early reflections) can be determined and then synthesized, there is some reason to expect the same realism with virtual sound. Moreover, as we come to better understand the stimulus control of auditory distance, it might be possible to vary the various cues, exaggerating some if necessary, so as to produce the desired variations in perceived distance.

\section{REFERENCES}

Amorim, M.-A., Glasauer, S., Corpinot, K., \& Berthoz, A. (1997). Updating an object's orientation and location during nonvisual navigation: A comparison between two processing modes. Perception \& Psychophysics, 59, 404-418.

Ashmead, D. H., DeFord, L. D., \& Northington, A. (1995). Contribution of listeners' approaching motion to auditory distance perception. Journal of Experimental Psychology: Human Perception \& Performance, 21, 239-256.

Begault, D. R. (1992). Perceptual effects of synthetic reverberation on three-dimensional audio systems. Journal of the Audio Engineering Society, 40, 895-904.

BEGAULT, D. R. (1994). 3-D sound for virtual reality and multimedia. New York: AP Professional.

Begault, D. R., \& Wenzel, E. M. (1993). Headphone localization of speech. Human Factors, 35, 361-376.

BööK, A., \& GÄRLING, T. (1981). Maintenance of orientation during locomotion in unfamiliar environments. Journal of Experimental Psychology: Human Perception \& Performance, 7, 995-1006.

Butler, R. A., Levy, E. T., \& NeFF, W. D. (1980). Apparent distance of sounds recorded in echoic and anechoic chambers. Journal of Experimental Psychology: Human Perception \& Performance, 6, 745750.

CARLILE, S. (1996). Virtual auditory space: Generation and applications, New York: Chapman \& Hall.

Coleman, P. D. (1963). An analysis of cues to auditory depth perception in free space. Psychological Bulletin, 60, 302-315.

Coleman, P. D. (1968). Dual role of frequency spectrum in determi- 
nation of auditory distance. Journal of the Acoustical Society of America, 44, 631-632.

CORLETT, J. T. (1986). The effect of environmental cues on locomotor distance estimation by children and adults. Human Movement Science, 5, 235-248

DURLACH, N. [I.] (1991). Auditory localization in teleoperator and virtual environment systems: Ideas, issues, and problems. Perception, 20, 543-554.

Durlach, N. I., Rigopoulos, A., Pang, X. D., Woods, W. S., KulkaRNi, A., Colburn, H. S., \& Wenzel, E. M. (1992). On the externalization of auditory images. Presence: Teleoperators \& Virtual Environments, 1, 251-257.

Fujita, N., LoOmis, J. M., Klatzky, R. L., \& GolledGe, R. G. (1990) A minimal model for dead-reckoning navigation: Updating the homing vector. Geographical Analysis, 22, 326-335.

Fukusima, S. S., Loomis, J. M., \& DA Silva, J. A. (1997). Visual perception of egocentric distance as assessed by triangulation. Journal of Experimental Psychology: Human Perception \& Psychophysics, 23, 86-100.

Gallistel, C. R. (1990). The organization of learning. Cambridge, MA: MIT Press.

GILKEY, R., \& ANDERSON, T. R. (1997). Binaural and spatial hearing in real and virtual environments. Hillsdale, $\mathrm{NJ}$ : Erlbaum.

Golledge, R. G., Loomis, J. M., Klatzky, R. L., Flury, A., \& Yang, X.-L. (1991). Designing a personal guidance system to aid navigation without sight: Progress on the GIS component. International Journal of Geographical Information Systems, 5, 373-395.

GuSKI, R. (1992). Acoustic tau: An easy analogue to visual tau? Ecological Psychology, 4, 189-197.

Haber, L., Haber, R. N., Penningroth, S., Novak, K., \& RadgowSKI, H. (1993). Comparison of nine methods of indicating the direction to objects: Data from blind adults. Perception, 22, 35-47.

IsRAEL, I., \& BERTHOZ, A. (1992). Representations of space and motion in man. In G. E. Stelmach \& J. Requin (Eds.), Tutorials in motor behavior (pp. 195-209). Amsterdam: Elsevier.

Kendall, G. S., \& MARTENS, W. L. (1984). Simulating the cues of spatial hearing in natural environments. In Proceedings of the 1984 International Computer Music Conference (pp. 111-125). San Francisco: Computer Music Association.

Klatzky, R. L., Beall, A. C., Loomis, J. M., Golledge, R. G., \& PHILBECK, J. W. (1998). Human navigation ability: Tests of the encoding-error model of path integration. Manuscript submitted for publication.

KRAMER, G. (1994). Auditory display: Sonification, audification, and auditory interface. Reading, MA: Addison-Wesley.

Laurent, M., \& Cavallo, V. (1985). Rôle des modalités de prise d'informations visuelles dans un pointage locomoteur [The role of visual input modality in a locomotor pointing task]. L'Année Psychologique, 85, 41-48.

LEE, D. N. (1990). Getting around with light or sound. In R. Warren \& A. H. Wertheim (Eds.), Perception \& control of self-motion (pp. 487505). Hillsdale, NJ: Erlbaum.

Little, A. D., Mershon, D. H., \& Cox, P. H. (1991). Spectral content as a cue to perceived auditory distance. Perception, 21, 405-41.6.

Loarer, E., \& Savoyant, A. (1991). Visual imagery in locomotor movement without vision. In R. H. Logie \& M. Denis (Eds.), Mental images in human cognition (pp. 35-46). The Hague: Elsevier.

LoOMIs, J. M. (1985). Digital map and navigation system for the visually impaired. Unpublished manuscript, University of California, Santa Barbara, Department of Psychology.

Loomis, J. M., Da Silva, J. A., Fujita, N., \& Fukusima, S. S. (1992). Visual space perception and visually directed action. Journal of Experimental Psychology: Human Perception \& Performance, 18, 906921.

Loomis, J. M., Golledge, R. G., Klatzky, R. L., Speigle, J. M., \& TIETZ, J. (1994). Personal guidance system for the visually impaired. In Proceedings of the ACM/Sigcaph Conference on Assistive Technologies (pp. 85-91). New York: Association of Computing Machinery.
Loomis, J. M., Hebert, C., \& Cicinelli, J. G. (1990). Active localization of virtual sounds. Journal of Acoustical Society of America, 88, 1757-1764.

Loomis, J. M., Klatzky, R. L., Golledge, R. G., Cicinelli, J. G., PelLEGRINO, J. W., \& FRY, P. A. (1993). Nonvisual navigation by blind and sighted: Assessment of path integration ability. Journal of Experimental Psychology: General, 122, 73-91.

Loomis, J. M., \& SoULE, J. I. (1996). Virtual acoustic displays for real and virtual environments. In Proceedings of the Society for Information Display 1996 International Symposium (pp. 965-968). San Jose, CA: Society for Information Display.

MaURer, R., \& Seguinot, V. (1995). What is modelling for? A critical review of the models of path integration. Journal of Theoretical Biology, 175, 457-475.

Mershon, D. H. (1997). Phenomenal geometry and the measurement of perceived auditory distance. In R. H. Gilkey \& T. R. Anderson (Eds.), Binaural and spatial hearing in real and virtual environments (pp. 257-274). Hillsdale, NJ: Erlbaum.

Mershon, D. H., Ballenger, W. L., Little, A. D., McMurtry, P. L., \& Buchanan, J. L. (1989). Effects of room reflectance and background noise on perceived auditory distance. Perception, 18, 403 416.

Mershon, D. H., \& Bowers, J. N. (1979). Absolute and relative cues for the auditory perception of egocentric distance. Perception, 8 , 311-322.

Mershon, D. H., \& KING, L. E. (1975). Intensity and reverberation as factors in the auditory perception of egocentric distance. Perception \& Psychophysics, 18, 409-415.

PHILBECK, J. W., \& LOOMIS, J. M. (1997). Comparison of two indicators of visually perceived egocentric distance under full-cue and reduced-cue conditions. Journal of Experimental Psychology: Human Perception \& Performance, 23, 72-85.

Philbeck, J. W., Loomis, J. M., \& Beall, A. C. (1997). Visually perceived location is an invariant in the control of action. Perception \& Psychophysics, 59, 601-612.

PICK, H. L., JR., \& Rieser, J. J. (1982). Children's cognitive mapping In M. Potegal (Ed.), Spatial abilities: Development and physiological foundations (pp. 107-128). New York: Academic Press.

Plenge, G. (1974). On the differences between localization and lateralization. Journal of the Acoustical Society of America, 56, 944-951.

Potegal, M. (1971). A note on spatial-motor deficits in patients with Huntington's disease: A test of a hypothesis. Neuropsychologia, 9 , 233-235.

Potegal, M. (1972). The caudate nucleus egocentric localization system. Acta Neurobiologiae Experimentalis, 32, 479-494.

Potegal, M. (1982). Vestibular and neostriatal contributions to spatial orientation. In M. Potegal (Ed.), Spatial abilities: Development and physiological foundations (pp. 361-387). New York: Academic Press.

Presson, C. C., \& Montello, D. R. (1994). Updating after rotational and translational body movements: Coordinate structure of perspective space. Perception, 23, 1447-1455.

RIESER, J. J. (1989). Access to knowledge of spatial structure at novel points of observation. Journal of Experimental Psychology: Learning, Memory, \& Cognition, 15, $1157 \cdot 1165$.

Rieser, J. J., Ashmead, D. H., Talor, C. R., \& Youngquist, G. A. (1990). Visual perception and the guidance of locomotion without vision to previously seen targets. Perception, 19, 675-689.

Rieser, J. J., Guth, D. A., \& Hill, E. W. (1986). Sensitivity to perspective structure while walking without vision. Perception, 15 , 173-188.

RIESER, J. J., \& Rider, E. A. (1991). Young children's spatial orientation with respect to multiple targets when walking without vision Developmental Psychology, 27, 97-107.

SCHIFF, W., \& OlDAK, R. (1990). Accuracy of judging time to arrival: Effects of modality, trajectory, and gender. Journal of Experimental Psychology: Human Perception \& Performance, 16, 303-316.

Shaw, B. K., McGowan, R. S., \& TuRveY, M. T. (1991). An acoustic variable specifying time-to-contact. Ecological Psychology, 3, 253-261. 
SHEELINE, C. W. (1983). An investigation of the effects of direct and reverberant signal interaction on auditory distance perception. Unpublished doctoral dissertation, Stanford University.

SPEIGle, J., \& LoOMis, J. M. (1993). Auditory distance perception by translating observers. In Proceedings of the IEEE Symposium on Research Frontiers in Virtual Reality (pp. 92-99). Washington, DC: IEEE.

Thomson, J. A. (1983). Is continuous visual monitoring necessary in visually guided locomotion? Journal of Experimental Psychology: Human Perception \& Performance, 9, 427-443.

Wenzel, E. M. (1992). Localization in virtual acoustic displays. Presence: Teleoperators \& Virtual Environments, 1, 80-107.
Wenzel, E. M., Arruda, M., Kistler, D. J., \& Wightman, F. L. (1993). Localization using nonindividualized head-related transfer functions. Journal of the Acoustical Society of America, 94, 11 1-123.

WightMAN, F. L., \& KisTLER, D. J. (1989). Headphone simulation of free-field listening: II. Psychophysical validation. Journal of the Acoustical Society of America, 85, 868-878.

(Manuscript received November 18, 1996; revision accepted for publication August 5, 1997.) 